\title{
Contralateral axillary lymph node metastasis and molecular changes in second primary breast cancer: a case report
}

\author{
Shuo Li ${ }^{1 \#}$, Fei Xie ${ }^{2 \#}$, Yan Li $^{1}$, Jue Wang ${ }^{1}$, Rui Chen ${ }^{1}$, Qian-Nan Zhu ${ }^{1}$, Xiao-Ming Zha ${ }^{1}$ \\ ${ }^{1}$ Breast Disease Department, the First Affiliated Hospital of Nanjing Medical University, Nanjing, China; ${ }^{2}$ Breast Disease Department, the Third \\ Affiliated Hospital of Soochow University, Suzhou, China \\ "These authors contributed equally to this work. \\ Correspondence to: Prof. Xiao-Ming Zha. Breast Disease Department, the First Affiliated Hospital of Nanjing Medical University, Nanjing, China. \\ Email: njzhaxm@njmu.edu.cn.
}

\begin{abstract}
Contralateral axillary metastasis (CAM) is rather rare in primary breast cancer. In this case, we present a 46-year-old female patient who underwent left breast-conserving surgery (BCS) and left axillary lymph node dissection (ALND). Two years later, an enlarged lymph node was found in her right axilla. Magnetic resonance imaging (MRI) of the breast displayed a left breast mass with multiple internal mammary lymph nodes and abnormal lymph nodes in the right axillary region. However, no abnormalities were found in the right breast. The left breast mass was diagnosed as invasive carcinoma by core needle biopsy. During the operation, we suggested that the contralateral lymph nodes were metastatic from the second primary breast cancer by preoperative $99 \mathrm{~m}$ Tc injection around the left breast. The patient underwent left mastectomy and right axillary lymph node dissection. The postoperative pathology was diagnosed as metachronous secondary primary left breast cancer, in which the initial presentation was lymph node metastasis to the contralateral axilla of the left breast. Therefore, we propose that CAM may be more common in second primary or recurrent breast cancer. It should be treated as locoregional extension. Preoperative lymph node markers are important to identify whether contralateral axillary lymph node metastasis occurs from a second primary breast cancer.
\end{abstract}

Keywords: Contralateral axillary node metastasis; breast cancer; preoperative lymph node markers; locoregional diffusion; case report

Submitted Jan 28, 2021. Accepted for publication Apr 12, 2021.

doi: $10.21037 /$ gs-21-137

View this article at: http://dx.doi.org/10.21037/gs-21-137

\section{Introduction}

An important prognostic feature of breast cancer is lymph node metastasis, and it has reference significance for treatment options. In a study of sentinel lymph node drainage patterns in untreated breast cancer patients, breast lymphatic drainage was as follows: ipsilateral axilla (92.3\%), intramammary $(21.1 \%)$, interpectoral $(2.1 \%)$, subclavian (2.6\%), and supraclavicular (0.4\%) (1). Contralateral axillary metastasis $(\mathrm{CAM})$ is uncommon in primary breast cancer, and the reported incidence is between $3.5 \%$ and $6 \%$. CAM can occur with primary breast cancer either synchronously or metachronously and can be the only site of metastasis. The management of these patients, especially those without distant metastasis, is controversial (2). The reported literature has considered CAM as stage IV disease, and there are no standard guidelines for CAM.

We present a case of a 46-year-old female who was diagnosed with metachronous secondary primary breast cancer with CAM, which is extremely rare. The histopathology and tumor characteristics of the metachronous secondary primary breast cancer with CAM in this case is that the tumor is multifocal invasive carcinoma with focal poorly differentiated neuroendocrine carcinoma. IHC demonstrated that the left breast cancer was negative for ER, PR, and HER2, which was similar to 

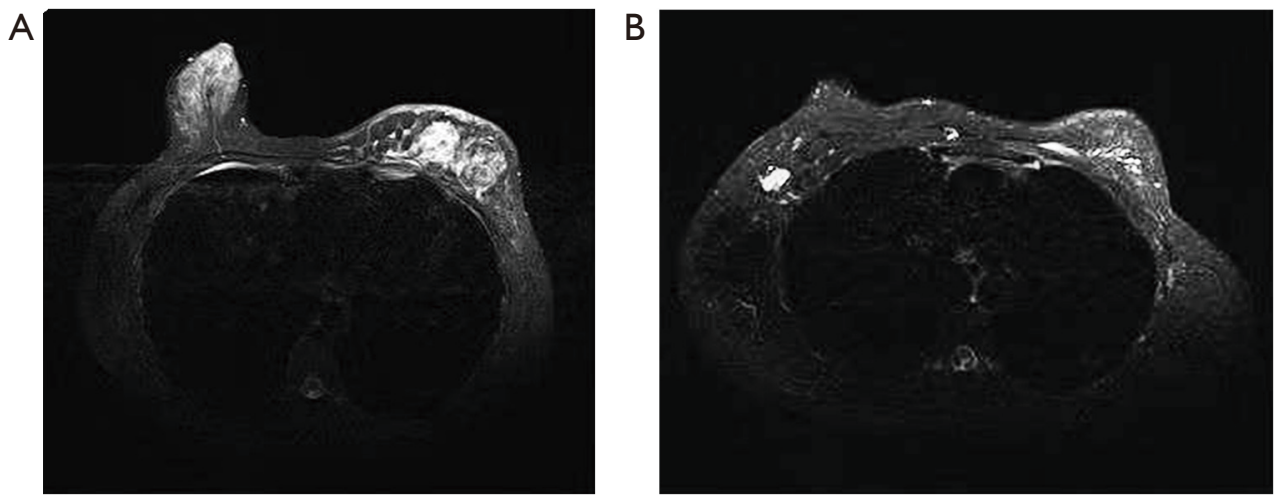

Figure 1 MRI images of the patient's breasts and axillary lymph nodes. (A) MRI of the breast revealed left breast cancer with multiple intrabreast metastases involving the nipple, areola, and skin, as well as subcutaneous cancerous lymphangitis, and numerous internal mammary lymph node metastases; (B) MRI of axillary lymph nodes revealed metastasis. MRI, magnetic resonance imaging.

the right metastatic axillary lymph nodes. We will describe how we confirmed the CAM of secondary primary breast cancer, along with our diagnosis, treatment, and follow-up.

We present the following article in accordance with the CARE reporting checklist (available at http://dx.doi. org/10.21037/gs-21-137).

\section{Case presentation}

In 2016, a 44-year-old female patient presented to the outpatient department of The First Affiliated Hospital of Nanjing Medical University (Jiangsu, China) due to a left breast lump. Physical examination revealed a painless, hard, and poorly mobile mass in the upper inner quadrant of the left breast, $2.0 \mathrm{~cm} \times 2.0 \mathrm{~cm}$ in size, without hydro derma, skin dimpling, nipple retraction, or nipple discharge. An ultrasound (US) displayed an irregularly shaped, lobulated, and calcified hypoechoic mass of $2.0 \mathrm{~cm} \times 1.2 \mathrm{~cm}$ without abnormal axillary lymph nodes, which was classified as category 5 based on the Breast Imaging Reporting and Data System (BI-RADS) (3). A molybdenum mammogram revealed a patchy, irregular, and slightly high-density mass with heterogeneous cluster calcification, $1.0 \mathrm{~cm} \times 1.8 \mathrm{~cm}$ in size. Core needle biopsy under ultrasonic guidance confirmed invasive carcinoma. After communicating with the patient about disease and surgical-related risks, she underwent left breast-conserving surgery (BCS) and left SLND. Intraoperative frozen pathology showed 1 of 4 lymph node metastases. She was suggested to undergo ALND. Postoperatively, histopathology revealed that the pathological diagnosis was invasive ductal carcinoma, with 1 of 10 lymph node metastases. Immunohistochemistry (IHC) was positive for estrogen receptor (ER) and progesterone receptor (PR) but negative for human epidermal growth factor receptor 2 (HER2). In postoperative adjuvant therapy, she was treated with 4 cycles of epirubicin $\left(75 \mathrm{mg} / \mathrm{m}^{2}\right)$ and cyclophosphamide $\left(600 \mathrm{mg} / \mathrm{m}^{2}\right)$ followed by 4 cycles of paclitaxel $\left(75 \mathrm{mg} / \mathrm{m}^{2}\right)$, and then underwent radiation therapy to the chest wall. Following the completion of chemotherapy, the patient has taken tamoxifen orally until now. Since the operation, she has been subsequently followed up every 6 months.

Two years later, in 2018, this patient came to our clinic for complaints of enlarged right axillary lymph nodes. MRI of the breast revealed left breast cancer with multiple intrabreast metastases involving the nipple, areola, and skin, along with subcutaneous cancerous lymphangitis, numerous internal mammary lymph node metastases, and 1 right axillary lymph node metastasis (Figure $1 A, B$ ). A $2.8 \mathrm{~cm} \times$ $2.7 \mathrm{~cm}$ irregularly shaped and mixed echo mass in the upper quadrant of the left breast and a $1.8 \mathrm{~cm} \times 1.0 \mathrm{~cm}$ enlarged lymph node in the right axillary region were displayed on US (Figure 2A,B). Physical examination revealed a posterior nipple mass in the left breast and right axillary lymph node enlargement. No abnormalities were found in the right breast. Other primary sites or any distant recurrences were not detected by systemic radiological examinations (brain MRI, chest CT, abdominal US, bone scintigraphy). The left breast mass was diagnosed as invasive carcinoma by core needle biopsy (Figure 3).

After consultation with the multidisciplinary team (MDT), which consisted of pathologists, oncologists, and 

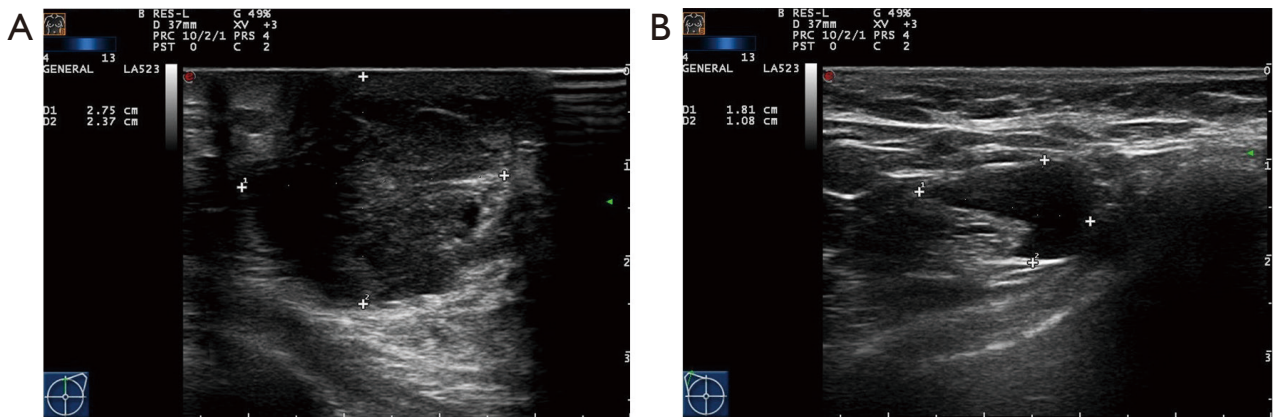

Figure 2 Ultrasound images of the patient's breast and axillary lymph nodes. (A) A $2.8 \mathrm{~cm} \times 2.7 \mathrm{~cm}$ irregularly shaped and mixed echo mass in the upper quadrant of the left breast was displayed on ultrasound; (B) $1.8 \mathrm{~cm} \times 1.0 \mathrm{~cm}$ enlarged lymph node in the right axilla was displayed on ultrasound.

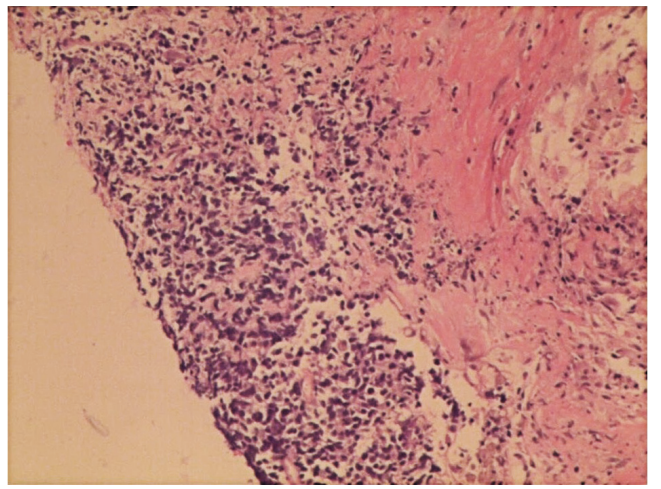

Figure 3 Pathological examination result (HE staining 200x). The left breast mass was diagnosed as invasive carcinoma by coarse needle puncture.

Radiologists, the patient underwent left mastectomy and right axillary lymph node dissection. To confirm whether right axillary lymph node metastasis occurred from the left breast or right occult primary cancer, we used $99 \mathrm{mTc}$ injected around the left breast mass and methylene blue next to the right areola prior to surgery. During the operation, the right axillary lymph nodes were found with both $99 \mathrm{mTc}$ and methylene blue, verifying that the right enlarged axillary lymph nodes were metastatic from the left breast. Pathology demonstrated multifocal infiltrating carcinoma in the left breast, with focal low differentiation neuroendocrine carcinoma involving the 4 quadrants and both vessels and nerves, and the largest quadrant had a maximum diameter of $5 \mathrm{~cm}$. Four of 23 right-sided axillary lymph nodes showed metastatic carcinoma. IHC demonstrated that the left breast cancer was negative for ER, PR, and HER2, which was similar to the right metastatic axillary lymph nodes. They were all different from the previous breast cancer on the left side. Therefore, we considered that this was the main site of right axillary lymph node metastases and diagnosed metachronous secondary primary cancer, in which the initial presentation was lymph node metastasis to the contralateral axilla of the left breast. The patient received 6 treatments of paclitaxel liposomes and capecitabine followed by radiation therapy to the right axilla. Follow-up surveillance imaging included MRI of the breast as well as systemic radiological examination. She remains disease-free at 20 months postoperation.

All procedures were in accordance with the ethical standards of the responsible committee on human experimentation (institutional and national) and with the Helsinki Declaration (as revised in 2013). This study was approved by the ethics and research committee of the First Affiliated Hospital of Nanjing Medical University. Informed consent was taken from all the patients.

\section{Discussion}

CAM is quite uncommon in the absence of metastatic disease elsewhere. Three possible sources should be considered: contralateral spread from the primary breast cancer; metastasis from an occult primary in the contralateral breast; and metastasis from an extramammary site. CAM may be more common in second primary and recurrent breast cancers as obstruction or damage of conventional axillary lymphatics may cause collateral circulation. This blockage may be caused by surgery and radiotherapy. In a meta-analysis of SLN biopsies in locally recurrent breast cancer by Maaskant-Braat et al., it was found that $43.2 \%$ of patients had abnormal drainage (4). Therefore, in second 
primary or recurrent breast cancer, the location of the sentinel lymph node becomes unforeseeable, confirming that after radiotherapy or previous surgery these drainage route changes may be obvious (5). In this case, the patient had previous left axillary lymph node dissection and radiotherapy. We hypothesized that CAM is caused by changes in lymphatic drainage and abnormal pathways and is secondary to lymphatic rather than blood-borne spread. Clinically, it is difficult to estimate CAM due to the lack of MRI to diagnose concealed contralateral tumors or failure to obtain adequate follow-up. However, CAM can be detected on preoperative lymphoscintigraphy and can be confirmed by lymph node biopsy and pathological diagnosis (6). IHC markers have been proven to usefully distinguish metastatic axillary lymph nodes from occult contralateral tumors. Here, we introduced a patient suffering from second primary left breast cancer and contralateral axillary lymph node metastasis. We confirmed this diagnosis by 99mTc and methylene blue, along with pathological IHC analysis. The IHC of the right lymph node was the same as that of the second breast cancer on the left, which was different from the primary tumor on the left.

It is rare if CAM has not metastasized to other distant organs, therefore, it is currently considered as M1 (stage IV) disease. Its management is considered complicated and is controversial. Nevertheless, some studies have reported that patients who develop CAM without other metastases to distant organs show longer overall disease-free survival than that of stage IV patients, which suggests that CAM should also be categorized as a local-regional disease (7). Therefore, treatment should intend to be curative rather than palliative. Moossdorff et al. performed a systematic review of CAM cases and reported an overall survival of $82.6 \%$ after an average follow-up of 50.3 months. CAM compares more favorably than concurrent stage IV disease (8). Chkheidze et al. retrospectively analyzed the medical records of 12 patients diagnosed with breast cancer and CAM, which, however, gives no evidence of any other distant metastases reflected by their clinical features, pathologic diagnoses, therapy, and data of follow-up. They suggested that isolated CAM without distant organ metastasis represents regional diffusion rather than distant metastasis (9). There have been other similar cases of CAM reported. Kinoshita $e t$ al. reported a 64-year-old female with metachronous second primary left occult breast cancer initially presenting as right axillary lymph node metastases who had received breast-conserving therapy for left breast cancer 4.5 years prior $(10,11)$. Christina et al. presented a patient who was diagnosed with invasive ductal carcinoma after left BCS and ALND at 9-year follow-up (12). Gingerich et al. showed that an 81-year-old woman, suffering from apparent second primary breast cancer, had a history of invasive ductal breast cancer 18 years before CAM (13). All 3 of these patients underwent radiation therapy and initial ALND, and contralateral axillary lymph node involvement did not show significant clinical signs until second primary ipsilateral breast carcinoma was found. However, they did not confirm that the metastatic lymph nodes were from primary breast lesions. Kim et al. found through preoperative FDG PET/CT and lymphoscintigraphy that the patient had second primary breast cancer, which was accompanied by contralateral axillary lymph node metastasis, emphasizing the usefulness of FDG PET/CT and lymphoscintigraphy to confirm the diagnosis (14).

However, all the above cases proposed that patients who underwent previous surgery or radiation therapy should receive curative treatments. In our case, the patient was suspected to have second primary left breast cancer with synchronous CAM by breast MRI $(6,15)$. We diagnosed second primary left breast cancer by needle biopsy and synchronous CAM by $99 \mathrm{mTc}$ and surgical pathology. We believed that CAM was local lymph node metastasis of the second primary left breast cancer as a result of changes in lymph node drainage. None of the evidence showed any disease of the right breast.

Because the possibility of contralateral occult breast cancer is very small, mastectomy is not recommended. We performed left mastectomy and right axillary lymph node dissection with curative intent.

\section{Conclusions}

We propose that CAM may occur in second primary or recurrent breast cancer because of previous surgery or radiation therapy. If changes in lymphatic drainage are suspected in patients with a history of breast cancer surgery, contralateral axillary lymph node metastasis should be considered. In patients with recurrent breast cancer, systemic examinations should be completed for the assessment of lymph node conditions, especially for the contralateral axillary lymph nodes. CAM could be detected through preoperative lymphoscintigraphy, lymph node biopsy, and pathological diagnosis. IHC markers have been shown to usefully distinguish metastatic axillary lymph nodes from occult contralateral tumors. We suggest that isolated CAM without distant organ involvement 
represents regional diffusion rather than distant metastasis and recommend specifying CAM as N3 instead of M1. We should treat CAM with curative intent rather than palliative intent, and axillary dissection offers good local control.

\section{Acknowledgments}

We thank Prof. Cong Wang for reviewing the pathology. Funding: None.

\section{Footnote}

Reporting Checklist: The authors have completed the CARE reporting checklist. Available at http://dx.doi.org/10.21037/ gs-21-137

Conflicts of Interest: All authors have completed the ICMJE uniform disclosure form (Available at http://dx.doi. org/10.21037/gs-21-137). The authors have no conflicts of interest to declare.

Ethical Statement: The authors are accountable for all aspects of the work in ensuring that questions related to the accuracy or integrity of any part of the work are appropriately investigated and resolved. All procedures were in accordance with the ethical standards of the responsible committee on human experimentation (institutional and national) and with the Helsinki Declaration (as revised in 2013). This study was approved by the ethics and research committee of the First Affiliated Hospital of Nanjing Medical University. Informed consent was taken from all the patients.

Open Access Statement: This is an Open Access article distributed in accordance with the Creative Commons Attribution-NonCommercial-NoDerivs 4.0 International License (CC BY-NC-ND 4.0), which permits the noncommercial replication and distribution of the article with the strict proviso that no changes or edits are made and the original work is properly cited (including links to both the formal publication through the relevant DOI and the license). See: https://creativecommons.org/licenses/by-ncnd/4.0/.

\section{References}

1. Wang W, Yuan P, Wang J, et al. Management of contralateral axillary lymph node metastasis from breast cancer: a clinical dilemma. Tumori 2014;100:600-4.

2. Magnoni F, Colleoni M, Mattar D, et al. Contralateral Axillary Lymph Node Metastases from Breast Carcinoma: Is it Time to Review TNM Cancer Staging? Ann Surg Oncol 2020;27:4488-99.

3. Estourgie SH, Nieweg OE, Olmos RA, Rutgers EJ, Kroon BB. Lymphatic drainage patterns from the breast. Ann Surg 2004;239:232-7.

4. Morcos B, Jaradat I, El-Ghanem M. Characteristics of and therapeutic options for contralateral axillary lymph node metastasis in breast cancer. Eur J Surg Oncol 2011;37:418-21.

5. Kinoshita S, Hirano A, Kobayashi S, et al. Metachronous secondary primary occult breast cancer initially presenting with metastases to the contralateral axillary lymph nodes: report of a case. Breast Cancer 2010;17:71-4.

6. Herold CI, Gaughan EM, Lamb CC, et al. Second primary ipsilateral breast cancer with contralateral axillary involvement: a case report and literature review. Clin Breast Cancer 2011;11:406-8.

7. Gingerich J, Kapenhas E, Morgani J, Heimann A. Contralateral axillary lymph node metastasis in second primary Breast cancer: Case report and review of the literature. Int J Surg Case Rep 2017;40:47-9.

8. Tasevski R, Gogos AJ, Mann GB. Reoperative sentinel lymph node biopsy in ipsilateral breast cancer relapse. Breast 2009;18:322-6.

9. Wellner R, Dave J, Kim U, et al. Altered lymphatic drainage after breast-conserving surgery and axillary node dissection: local recurrence with contralateral intramammary nodal metastases. Clin Breast Cancer 2007;7:486-8.

10. Lizarraga IM, Sugg SL, Weigel RJ, et al. Review of risk factors for the development of contralateral breast cancer. Am J Surg 2013;206:704-8.

11. Trifirò G, Ravasi L, Paganelli G. Contralateral or bilateral lymph drainage revealed by breast lymphoscintigraphy. Eur J Nucl Med Mol Imaging 2008;35:225-9.

12. Kim SJ, Moon WK, Cho N, et al. The detection of recurrent breast cancer in patients with a history of breast cancer surgery: comparison of clinical breast examination, mammography and ultrasonography. Acta Radiol 2011;52:15-20.

13. Kroon BB, Hoefnagel CA, Valdés Olmos RA, et al. Regional lymph nodes at a distance. Ned Tijdschr Geneeskd 2008;152:1997-2000.

14. Roumen RM, Kuijt GP, Liem IH. Lymphatic mapping and sentinel node harvesting in patients with recurrent breast 
cancer. Eur J Surg Oncol 2006;32:1076-81.

15. Nishimura S, Koizumi M, Kawakami J, et al. Contralateral axillary node metastasis from recurrence after conservative breast cancer surgery. Clin Nucl Med 2014;39:181-3.

(English Language Editor: C. Betlazar-Maseh)

Cite this article as: Li S, Xie F, Li Y, Wang J, Chen R, Zhu QN, Zha XM. Contralateral axillary lymph node metastasis and molecular changes in second primary breast cancer: a case report. Gland Surg 2021;10(4):1547-1552. doi: 10.21037/gs-21-137 